\title{
Body-Surface Atrial Signals Analysis Based on Spatial Frequency Distribution : Comparison Between Different Signal Transformations
}

\author{
Olivier Meste ${ }^{1}$, Stef Zeemering ${ }^{2,4}$ Joël Karel $^{5}$, Theo Lankveld ${ }^{3,4}$, Ulrich Schotten ${ }^{2,4}$, Harry Crijns ${ }^{3,4}$, \\ Ralf Peeters ${ }^{5}$, Pietro Bonizzi ${ }^{5}$ \\ ${ }^{1}$ Laboratoire I3S, UNSA-CNRS, Nice, France \\ ${ }^{2}$ Department of Physiology, Maastricht University Medical Center, Maastricht, The Netherlands \\ ${ }^{3}$ Department of Cardiology, Maastricht University Medical Center, Maastricht, The Netherlands \\ ${ }^{4}$ Cardiovascular Research Institute Maastricht (CARIM), Maastricht University, The Netherlands \\ ${ }^{5}$ Department of Data Science and Knowledge Engineering, Maastricht University, Maastricht, The \\ Netherlands
}

\begin{abstract}
In contrast to electrograms, Body-Surface Potential Mapping (BSPM) records the global atrial activity, at the expenses of a lower spatial accuracy. The aim of this study is to investigate whether BSPM recordings can discriminate persistent patients undergoing electrical cardioversion, based on the body-surface normalized AF spatial frequency distribution. High-density BSPMs (120 anterior, 64 posterior electrodes) were recorded in 63 patients with persistent AF. For each patient and electrode recording, the frequency content of $A F$ was analyzed on the raw signal, and also by means of the normalized correlation function, and by Singular Spectrum Analysis (SSA). In order to compare the body-surface spatial distributions of AF frequency in all patients, these distributions were first normalized, before performing statistical analysis.

We found that the distribution of AF frequency on the body-surface, and its interpretation, are strongly dependent on the specific method employed. Moreover, the estimated body-surface AF frequency was greater over the central posterior and the right anterior BSPM locations. Finally, SSA-based decomposition followed by frequency analysis could discriminate AF patients recurring 4 to 6 weeks after electrical cardioversion from those who did not, based on the frequency content in the proximity of VI.
\end{abstract}

\section{Introduction}

Atrial fibrillation (AF) dominant frequency has been shown to be a relevant feature in both determining the stage of AF, and in patient's characterization. As already noticed in [1], the noninvasive analysis of AF by means of Body-Surface Potential Mapping (BSPM), allows the measurement of the AF spatial frequency distribution with still good accuracy with respect to the same quantity measured invasively. This distribution informs us on the propagation of AF waveforms over the atria with the potential of guiding radiofrequency ablation procedures. Considering a single frequency value for global AF characterization may not suffice for further analysis such as discriminating between patients showing AF recurrence (R) or nonrecurrence (NR) after electrical cardioversion.

It has been shown in [2] that multidimensional autocorrelation function enables such discrimination based on short and long term features of the multidimensional AF vector. In addition, this discrimination has also been performed successfully at the level of individual electrodes by using similar method [3], and showing that spatial dimension plays an important role in this process. In order to test the power of AF frequency distribution to discriminate $\mathrm{R}$ from NR patients, the frequency content recorded by each electrodes was processed by using different methods. In particular, the approach proposed in [3] and was compared to regular spectral analysis based on windowed Fast Fourier Transform (FFT) and Singular Spectrum Analysis (SSA) [4]. Results showed that, thanks also to a patientspecific frequency normalization, specific regions on the torso differ from others in terms of frequency distribution, and that a gradient in magnitude exists instead of a frequency one.

\section{Methods}

\subsection{BSPM data and pre-processing}

BSPMs were recorded in 63 patients in persistent AF using a custom configuration of 184 leads with 120 anterior and 64 posterior leads (ActiveTwo BSM Panels Carbon Electrodes, Biosemi B.V., The Netherlands). All pa- 
tients underwent electrical cardioversion. After a blanking period from 4 to 6 weeks after procedure, 32 patients showed AF recurrence (labeled R) and 31 remained in sinus rhythm (labeled NR). ECGs were sampled at $256 \mathrm{~Hz}$. A one-minute segment was selected for each subject, lowquality leads were excluded (low signal-to-noise ratio, poor electrode contact, motion artefacts), and Wilsons Central Terminal was subtracted in line with conventional ECG analysis. After band-pass filtering the signals between 1 and $100 \mathrm{~Hz}$ (3rd order Chebyshev), QRST cancellation was performed using an adaptive singular value decomposition method, inspired by the approach in [5], with multiple QRST window templates defined using hierarchical clustering. The extracted atrial signals were postfiltered with a zerophase notch filter at $50 \mathrm{~Hz}$ to suppress power line noise, and with a $3 \mathrm{~Hz}$ zero-phase highpass filter (3rd order Chebyshev) to remove low-frequency residuals not related to (persistent) AF.

\subsection{AF vector Autocorrelation}

In a previous study [3], we showed that the multivariable autocorrelation function stems from the contribution of monodimensional autocorrelation functions computed from individual electrode recordings. From that observation, it comes that the AF frequency content of a patient can be extracted for each electrode by computing the FFT of the corresponding monodimensional autocorrelations (producing the results named "Monocorr" in this study). Furthermore, the value of the first max of the monodimensional autocorrelation function plays also a crucial role in the characterization of the AF [2]. Thus, it is quite intuitive to compute the time lag of this value, assumed to be related to the AF cycle length, and calculate a second estimate of the AF frequency for each electrode (named "Monolag").

\subsection{Singular Spectrum Analysis}

The singular spectrum analysis is based on the embedding of the time series $x(n)$, associated to a recorded ECG lead, in a vector of dimension $M$. In this study,we fixed $M$ to be equal to 150 samples, according to the $\mathrm{AF}$ mean short time behavior observed in [2]. The embedding procedure forms $K=N-M+1$ lagged vectors $\boldsymbol{x}_{i}=(x(i), \ldots, x(i+M-1))^{T}$. A matrix $\boldsymbol{X}$ is then generated as $\boldsymbol{X}=\left[\boldsymbol{x}_{\boldsymbol{1}}, \boldsymbol{x}_{\mathbf{2}}, \ldots, \boldsymbol{x}_{\boldsymbol{M}}\right]$, such that this is a Hankel matrix. The singular value decomposition (SVD) of $\boldsymbol{X}$ is then computed, such that $\boldsymbol{X}=\boldsymbol{U} \boldsymbol{D} \boldsymbol{V}^{T}$. The matrix $\boldsymbol{X}$ is then reconstructed by the SVD-based rank two approximation $\boldsymbol{X} \approx \sigma_{1} \boldsymbol{u}_{1} \boldsymbol{v}_{1}^{T}+\sigma_{2} \boldsymbol{u}_{2} \boldsymbol{v}_{2}^{T}$. The $\sigma, \boldsymbol{u}$ and $\boldsymbol{v}$ values stands for the two highest singular values and the corresponding right and left eigenvectors, respectively. The rank two approximation was selected in order to minimize the approximation error, while taking into account the complementarity of $\sin$ and $\cos$ functions (expected to be reflected in the first two principal components, respectively). The last step of the SSA consists in averaging the diagonal of the reconstructed matrix to provide the approximated time series $x(n)$ (see [6] for a complete description of the procedure and possible extensions). Finally, an FFTbased spectral analysis was applied to the reconstructed series.

\section{Results and Analysis}

The four frequency analyses were performed on the two groups of subjects, according to the R and NR classification. Thus four estimations (FFT, Monocorr, Monolag, SSA) of the frequency with the highest power were provided for each patient and for each electrodes recording. For a given patient, the dominant frequencies associated to each electrode were normalized in the range [0-1]. This normalization is crucial to account for inter-patient variability when assessing the locations of the highest and lowest frequency value (regardless their absolute values). Figures (1), (2), (3), (4) show the median values of the dominant frequencies of all patients, computed over each electrode. The black circles stand for the electrode locations and the red ones for the lead V1 position. A Man-Whitney $\mathrm{U}$ test was performed on the $\mathrm{R}$ and NR patients populations for stratification purposes, and applied individually to each electrode. A significant $(p<0.05)$ difference is highlighted by a bold black circle. It should be noted that no corrections were applied for multiple comparisons.

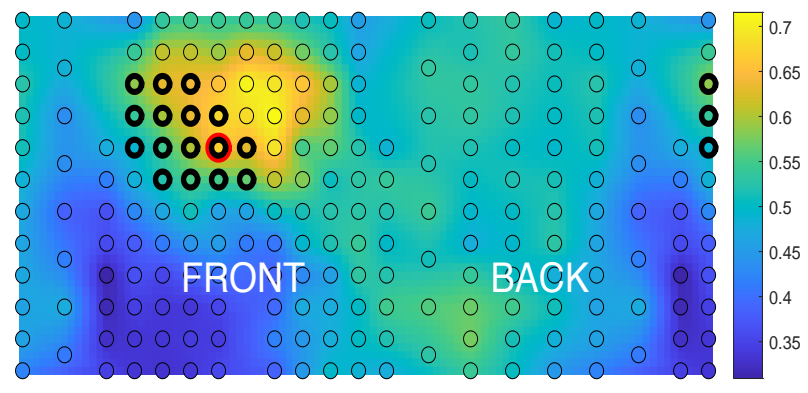

Figure 1. Median normalized dominant frequencies (Monocorr) for all patients and for all electrodes. See text for details.

As it can be noticed from the figures, all maps exhibit similar results, with the highest frequencies localized on the right anterior side of the torso. The corresponding location on the heart surface is the right atrium. In addition, the FFT results seem to be the more scattered, missing the regional homogeneity of frequency content. Thus, an additional criterion is needed to compare the performance of the three methods left.

This criterion could be the frequency resolution associated with each method and its influence over the frequency 


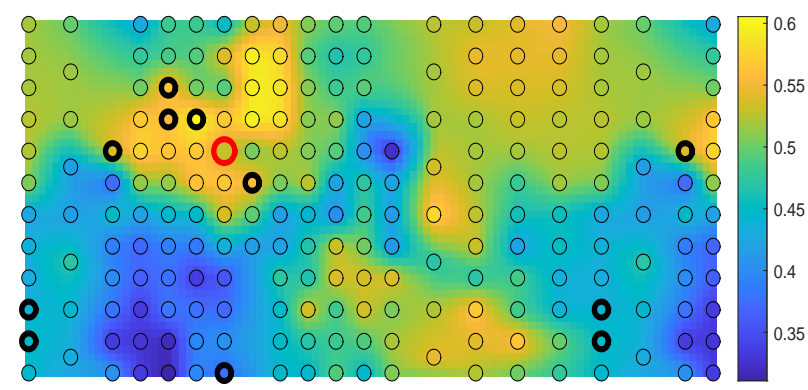

Figure 2. Median normalized dominant frequencies (FFT) for all patients and for all electrodes. See text for details.

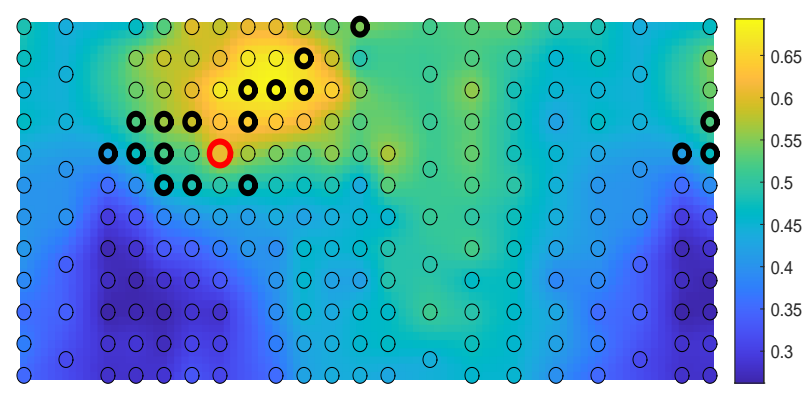

Figure 3. Median normalized dominant frequencies (Monolag) for all patients and for all electrodes. See text for details.

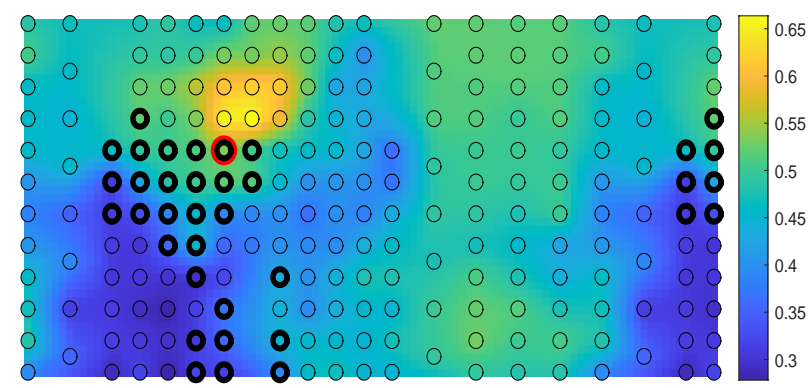

Figure 4. Median normalized dominant frequencies (SSA) for all patients and for all electrodes. See text for details

map interpretation. This is illustrated with a single NR patient exhibiting a clear AF pattern. In Fig. (6), and (8) regions of constant frequencies are clearly identifiable. Such properties have been already observed in [1], and can be related to dominant frequencies computed over electrograms recorded in various regions of the heart. In contrast, in Fig. (5), and (7) a frequency gradient is visible and can be explained by the low frequency resolution of the corresponding frequency estimators. It can be easily observed that a gradient in the magnitude of the dominant frequency can be easily converted (and mistaken) into a gradient in frequency because of a limited resolution. This example shows that SSA and FFT seem to provide more accurate estimates, although as shown above the FFT globally per- formed badly in term of AF patients discrimination.

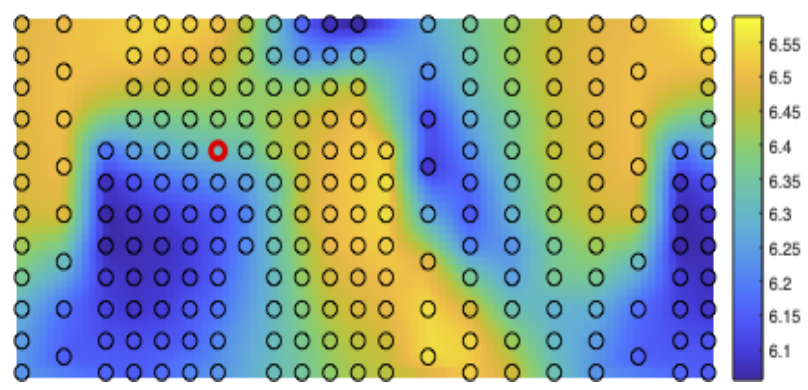

Figure 5. Normalized dominant frequencies (Monocorr) for a NR patient and for all electrodes.

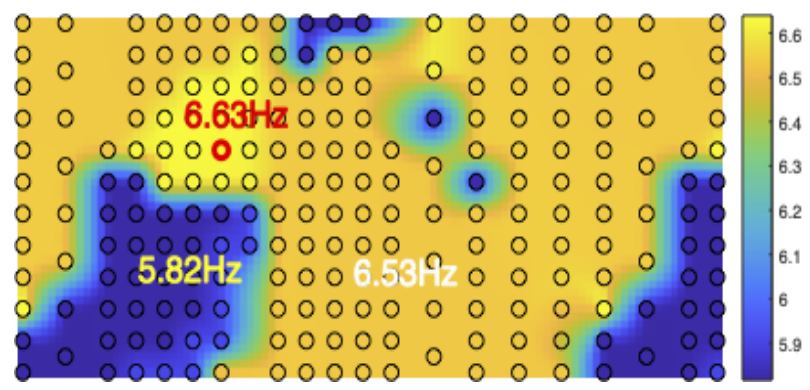

Figure 6. Normalized dominant frequencies (FFT) for a NR patient and for all electrodes.

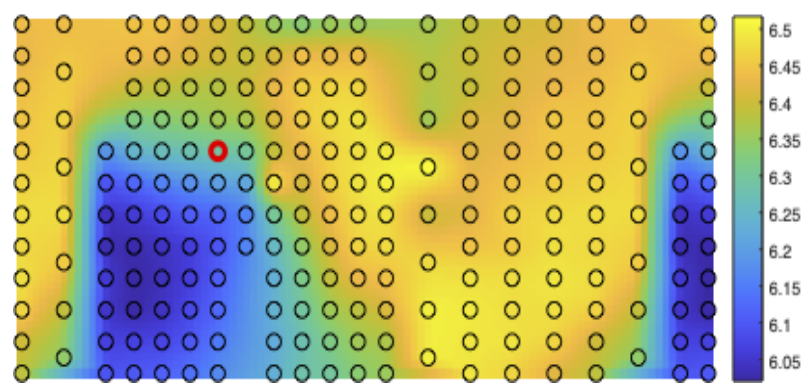

Figure 7. Normalized dominant frequencies (Monolag) for a NR patient and for all electrodes.

\section{Conclusions}

This study compared different (AF) dominant frequency estimation methods for the discrimination of $\mathrm{R}$ and $\mathrm{NR} A \mathrm{AF}$ patients. It is shown that this comparison can be performed at the level of each electrodes. As expected the dominant frequency varies along the body surface in accordance to the heart electrophysiology during AF. The dominant AF frequency is on average localized at the right anterior side of the torso corresponding to the right atrium. This results are consistent with clinical outcomes using electro- 


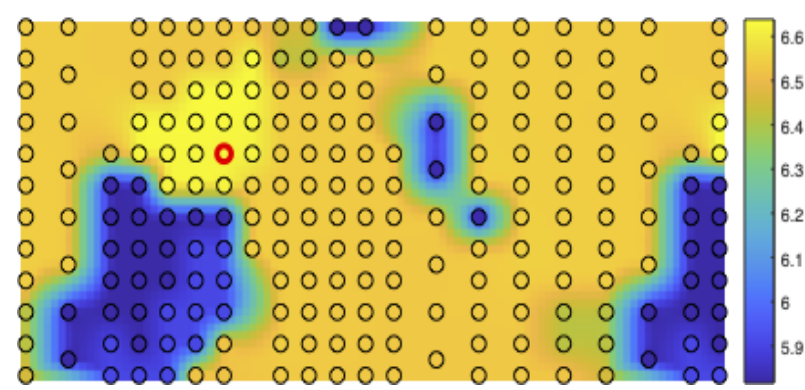

Figure 8. Normalized dominant frequencies (SSA) for a NR patient and for all electrodes.

grams. Finally, it seems that the SSA-based approach allows a plausible interpretation and discrimination between NR and R patients together with the generation of an accurate frequency mapping. These results have been obtained thanks to a proper normalization of the frequency range applied to each patient.

\section{References}

[1] Guillem MA, Andreu MC, Millet J, Arenal A, FernandezAviles F, Jalife J, Atienza F, Berenfeld O. Noninvasive localization of maximal frequency sites of atrial fribrillation by body surface potential mapping. Circ Arrhythm Electrophysiol. 2013; 6:294-301.
[2] Bonizzi P, Meste O, Zeemering S, Karel J, Lankveld T, et al.. A novel framework for noninvasive analysis of shortterm atrial activity dynamics during persistent atrial fibrillation. Medical and Biological Engineering and Computing, Springer Verlag, 2020;58:1933-1945.

[3] Meste O, Zeemering S, Karel J, Lankveld T, Schotten U, Crijns H, Peeters R, Bonizzi P. Use of normalized correlation function to discriminate outcome of persistent patients undergoing electrical cardioversion. Conf Proc Comp in Card, 2020; Vol. 47

[4] Vautard R, Ghil M. Singular spectrum analysis in nonlinear dynamics, with applications to paleoclimatic time series. Physica D, 1989, 35, 3: 395-424.

[5] Alcaraz R, Rieta JJ. Adaptive singular value cancellation of ventricular activity in single-lead atrial fibrillation electrocardiograms. Physiol Meas, 2008;29:1351-1369.

[6] Bonizzi P, Karel JMH, Meste O, Peeters RLM. Singular spectrum decomposition: A new method for time series decomposition. Advances in Adaptive Data Analysis, 2014, Vol. 6, 4: 34 pages.

Address for correspondence:

Pr. Olivier MESTE

Laboratoire I3S - CNRS - UNS

2000 route des Lucioles

06903 Sophia Antipolis cedex, FRANCE

E-mail address: olivier.meste@univ-cotedazur.fr 